\title{
IL MONDO OFFESO DI ELIO VITTORINI
}

\author{
Giovanna Armellin Secchi
}

\begin{abstract}
The purpose of this literary study is to contribute to the knowledge about Elio Vittorini's most important themes, by the analysis of his short stories and novels. Vittorini (19081966), a self-educated writer, considered the predecessor of Neorealism, was an engaged intellectual. He wrote to denounce the suffering of many people because of social injustice, that means, an offended world - "un mondo offeso"-.
\end{abstract}

Elio Vittorini nasce in Sicilia nel 1908, vive quindi all'ombra delle guerre e dei problemi inerenti ad esse.

Questo scrittore, considerato da molti come l'iniziatore del Neorealismo, rappresenta la figura dell'intellettuale "impegnato" che si fa portavoce dei disagi del "mondo offeso", il mondo degli umili, degli oppressi e dell'ingiustizia per la quale " Un uomo ride e un altro piange".

Lo scrittore, attraverso le sue opere rivela le pene di tutti gli uomini, in un mondo alienato dall'ingiustizia e dall'oppressione che stabiliscono fra loro un'incomunicabilità e corrompono la stessa persona umana.

La commossa fraternità, che traspare in tutti i suoi testi, per l'uomo che soffre riflette il senso di disagio che prova buona parte della gioventù, diventa perciò interprete di sentimenti comuni a tutti in questo periodo eccezionale.

Vittorini apre la sua parabola letteraria, potremmo dire la sua missione letteraria con la fatica dell'autodidatta.

Lo scrittore comincia a dare la sua collaborazione alla letteratura e ai problemi del suo tempo scrivendo su "Solaria" la rivista fiorentina che più vivacemente esprime l'insofferenza per il ghetto culturale che il fascismo ha creato.

In queste pagine Vittorini si forma scrittore, fa la sua prima esperienza letteraria che lo stacca dalla prosa d'arte per orientarlo verso il racconto ed il romanzo.

Il garofano rosso pubblicato a puntate su "Solaria" e sospeso quasi subito dalla censura fascista, lo segnala come scrittore della nuova generazione.

I letterati italiani del momento si snervano in discussioni apparentemente libere, ma in realtà asservite alla dittatura fascista. 
Così in un clima di intimidazione culturale ci si stordisce nella retorica fascista. La verbosità dannunziana crea un ambiente di entusiasmo fittizio, dando voce al mito del super uomo di Nietsche a cui la dittatura dà concretezza.

In questa atmosfera Vittorini comincia a esprimere la propria linea narrativa.

Nel 1931 esce la sua prima opera: Piccola borghesia che già nel titolo indica una spiccata inclinazione per certi contenuti umano-sociali una voluta attenzione a cogliere, al modo neorealistico, il disagio e la tristezza di un certo ambiente, di una certa condizione.

I sei racconti che compongono il volume hanno uno stile che ritornerà nelle opere seguenti: la descrizione sospesa tra il dato lirico e quello realistico, attenta ad offrire una rappresentazione della vita non accademica, ma reale.

Vittorini rivela in questi brevi racconti, con crudezza nella descrizione, di essere alla ricerca di una propria dimensione narrativa e sperimenta in essi diverse possibili soluzioni. Il libro oggi è appena ricordato, ma presenta già i caratteri fondamentali del mondo di Vittorini: profondo sentire umano impoverito però dalla dimensione quasi istintiva in cui sono colti i personaggi. Lo stile alterna pagine crudamente realistiche a pagine di contenuta liricità.

Qualche anno dopo esce il primo romanzo di Vittorini: Il garofano rosso, sospeso in seguito dalla censura fascista. Il romanzo impostato su di una visione paganeggiante della vita, è documento del clima di violenza che sconvolge l'Italia nel 1924 per l'uccisione di Matteotti e del sentimento confuso di rivolta contro la società costituita che anima i giovani.

In questo ambiente in fermento l'autore costruisce la vicenda del protagonista che alterna la sua violenta esperienza di giovane fascista dalle idee poco chiare, a quella sentimentale.

La politica come l'amore è per il protagonista un'avventura da vivere intensamente e, per essere tale non può essere vissuta che in contrasto con le convenzioni e le regole costituite. L'amore che diventa il motivo dominante della seconda parte della narrazione, è ridotto a dato materiale, istintivo. Ogni attesa della giovinezza, in queste pagine, si esaurisce in un'emozione momentanea.

Il disordine morale non può soddisfare l'uomo e questo lo sperimenta lo stesso protagonista quando esprime, molto fuggevolmente il vuoto che la vita ridotta a dimensioni istintive, genera nell'individuo: "Ci si inganna, ci si stordisce a vicenda, e si dice che è la vita!... Che odiosa rettorica!"(Vittorini 1981: 190).

La conoscenza dell'inglese permette a Vittorini di avvicinarsi alle opere degli scrittori più rappresentativi del realismo americano. Lui cerca nella letteratura americana la risposta alle proprie esigenze di scrittore e di uomo.

Sebbene il momento politico tenda a chiudere l'Italia in un ghetto, Vittorini inizia il lavoro di traduttore della letteratura americana e diviene ponte tra la mentalità americana e quella italiana.

Questa scuola di stile e di mentalità degli scrittori d'oltreoceano lo rende più attento alle "cose", ma non lo priva di una fisionomia personale che trasforma le traduzioni in vere e proprie rielaborazioni.

Si opera così quel rinnovamento del gusto che esploderà nel neorealismo dell'immediato dopoguerra e ci si orienta ad uno sperimentalismo narrativo per una via fino ad ora ignorata. 
Proprio in Saroyan, uno scrittore armeno immigrato in America, Vittorini scopre quel procedimento tecnico che adotta nella stesura di Conversazione in Sicilia

Egli giudica che:

(...) non ha fatti da narrare, situazioni da svolgere, ma "cose" da dire e i personaggi non lo interessano che come simboli delle cose che ha da dire. La sua ispirazione è lirica. La sua composizione è uno sfogo lirico. (Pazzaglia 1972: 1346)

Vittorini è siciliano e, anche se la maggior parte delle sue opere è ambientata ai margini di Milano e nell'Italia settentrionale, lo sentiamo veramente vivo quando ritorna nell'ambiente della sua terra natale. I personaggi riflettono bene l'anima ardente e contenuta, al tempo stesso, degli isolani ed è caratteristica quell'atmosfera di tristezza che invade l'ambiente e incombe fatalmente sulla vita di tutti.

Conversazione in Sicilia narra di un viaggio dell'autore in Sicilia per rivedere sua madre dopo quindici anni di assenza. In realtà egli va alla riscoperta della sua terra e della sua infanzia. In due giorni di permanenza in una casa dove non era mai vissuto, vicino ad una donna -sua madre- che ricordava confusamente, ha modo di ricostruire non senza sorprese, la sua infanzia infelice.

La madre che vive come può dopo l'abbandono del marito, lo conduce con sé nel vicinato dove ella presta il suo aiuto di infermiera. Il protagonista viene così crudamente a contatto col male, col dolore: vede per la prima volta casupole e tuguri dove fame e malattia sono accettati come mali inerenti alla condizione umana.

Il problema della sofferenza si presenta angoscioso alla sua mente; vuol sapere da sua madre che cosa ne pensa di tutta quella gente inebetita dalla miseria ed ella risponde "Penso che forse non potranno pagarmi.'(Vittorini 1978a: 101).

Questa espressione amara, quasi cinica, se chi la pronuncia non fosse a sua volta immersa nello stesso squallore morale e materiale, questa secca risposta che rivela, secondo Vittorini, il modo più comune di sentire le pene altrui, conferma in lui una convinzione: il vivere dell'uomo ha un solo senso, la non-speranza.

In una divisione quasi elementare egli divide la società in due classi: gli uomini e i non-uomini.

Ogni uomo è malato, una volta, nel mezzo della sua vita, e conosce questo estraneo che è il male, dentro di lui, l'impotenza sua con questo estraneo : può comprendere il proprio simile ... Ma forse non ogni uomo è uomo, e non tutto ill genere umano è genere umano. Un uomo ride e l'altro piange. Tutti e due sono uomini: anche quello che ride è stato malato ed è malato; eppure egli ride perché l'altro piange. Non ogni uomo allora è uomo. Uno perseguita e l'altro è perseguitato; e genere umano non è tutto il genere umano, ma quello soltanto del perseguitato. Uccidete un uomo: egli sarà più uomo. E così è più uomo un malato, un affamato, è più genere umano il genere umano dei morti di fame. (Vittorini 1978a: 100)

A Vittorini appare epica la condizione dell'uomo che soffre con una sorta di stoicismo fatto di silenzio, al modo dei personaggi di Camus che accettano di vivere giorno per giorno senza pensare al futuro. 
È opportuno ricordare che quando stende Conversazione in Sicilia sono i giorni della guerra di Spagna ed egli è colpito dalla sorte dell'uomo in balia della miseria e della morte che ne spezzano la resistenza fisica, ma anche dell'oppressione politica e della violenza che lo schiantano moralmente.

Non meno significativi gli incontri con personaggi emblematici che propongono al protagonista diverse soluzioni per alleviare la sofferenza nel mondo e quello di Silvestro con il fratello Liborio morto da pochi giorni in guerra e venuto a riposarsi nel cimitero vicino a casa sua.

In chiave lirica Vittorini vede la sua stessa isola interprete della tragedia dell'uomo:

Era notte sulla Sicilia e la calma terra: l'offeso mondo era coperto di oscurità; gli uomini avevano lumi accanto chiusi con loro nelle stanze, e i morti, tutti gli uccisi, si erano alzati a sedere sulle loro tombe. Meditavano. Io pensai e la grande notte fu in me, notte su notte. (Vittorini 1978a: 215)

E in una serie di immagini sottilmente evocate tra il simbolo e la realtà, Vittorini richiama crudamente le miserie del mondo senza luce alcuna di speranza. La conclusione non può essere che il pianto sconsolato dell'autore su di un mondo di sofferenza chiuso in se stesso. Conclusione amara di chi non sa far altro che prendere atto della miseria dell'uomo, incapace di scoprire gli elementi di riscatto che il dolore offre.

In Conversazione in Sicilia non si può rintracciare una trama: il discorso sfuma continuamente nel simbolo.

Vi si rintracccia invece immediatamente un' atmosfera dominata dalla fatalistica ed antica tristezza isolana, dall'incombente motivo del problema del male che offende il mondo e pesa su di un popolo gravato come da una sua secolare disperazione. (Scaramucci 1956: 245)

Il problema della sofferenza dell'uomo impegna Vittorini: purtroppo il suo discorso si esaurisce in una constatazione di fatto che preclude ogni speranza.

Vittorini aderisce all'ideologia comunista ma, come Pavese, è un comunista insoddisfatto, sempre alla ricerca di qualcosa che possa rispondere esaurientemente a tanti problemi umani e sociali che il solo elemento economico non può risolvere.

Vittorini, nel dopoguerra, diventa un poco il simbolo della nuova cultura. I giovani guardano a lui. Si comincia a parlare del neorealismo, cioè di una ricerca nuova della realtà e di un nuovo modo di presentare la realtà.

Vittorini nel 1945 tenta un proprio giornale: Il Politecnico e ne esprime i motivi: suscitare un dialogo tra cultura e politica perché questa non rimanga pura retorica, affrontare fattivamente la crisi che già mina l'ideologia comunista e promuovere un'apertura decisa verso la letteratura internazionale.

Nel primo numero del suo giornale Vittorini fa un bilancio della guerra:

Per un pezzo sarà difficile dire se qualcuno o qualcosa abbia vinto in questa guerra. Ma certo vi è tanto che ha perduto e che si vede come ha perduto! I morti, se li contiamo, sono più bambini che soldati, le macerie sono di città che avevano venticinque secoli di vita, di case, di biblioteche, di monumenti, di 
cattedrali, di tutte le forme per le quali è passato il progresso civile dell'uomo, e i campi in cui si è sparso più sangue si chiamano: Mathausen, Dakau, Maidanek, Buchenwald. (Vittorini 1945n.I, 21 settembre 1945)

Così Vittorini, sofferente per un assillo che gli urge dentro, continua a tenere fede all'impegno di quella problematica umana che era già un perno della precedente narrativa. Egli è convinto che il compito dello scrittore non può risolversi che in una continua ricerca della verità anche in quelle manifestazioni che sembrano estranee all'esercizio letterario. Anzi è proprio questa sua inquietudine di ricerca che mette in allarme il partito comunista. Nel 1947, a solo due anni dall'inizio di Politecnico, Togliatti ordina la soppressione della rivista che sconfina dalle linee del partito.

La vitalità di Vittorini nel dare incremento ad una letteratura impegnata si rivela anche nel romanzo del 1945 dove il motivo fondamentale è la discriminazione dell'umanità: Uomini e no. Il titolo stesso lo denuncia.

L'uomo, si dice e noi pensiamo a chi cade, a chi è perduto, a chi piange, a chi ha fame, a chi ha freddo, a chi è perseguitato, a chi viene ucciso. Pensiamo all'offesa che gli è stata fatta, alla dignità di lui. Anche a tutto quello che in lui è offeso, e ch'era, in lui, per renderlo felice. Questo è l'uomo... (Vittorini 1978c: 157)

Uomini e no è il primo romanzo sulla resistenza partigiana. È un itinerario di guerra spoglio di tutte le strutture romantiche, crudo nell'evidenza dei fatti, dei dialoghi, nell'espressione dei sentimenti e delle emozioni a volte appena accennate. La guerra è presentata in queste pagine nella sua crudele evidenza di sterminio e di lotta.

Siamo a Milano durante i tragici mesi dell'oppressione tedesco-fascista. I fatti narrati pochi e atroci; significative, ma senza risposta le considerazioni personali dell'autore che porta l'attenzione di chi legge su interrogativi fondamentali:

Bisogna che gli uomini possano essere felici. Ogni cosa ha un senso solo perché gli uomini siano felici. Non è solo per questo che le cose hanno senso? (Vittorini 1978c: 13)

Anche uccidere e farsi uccidere acquista un significato solo in vista di un'umanità più felice. È questo il pathos della lotta partigiana che si svolge a Milano nel turbinoso inverno del 1944.

Perché vivevano come animali inseguiti e ogni giorno esponevano la loro vita?

Perché dormivano con la pistola sotto il cuscino? Perché lanciavano bombe?

Perché uccidevano? (Vittorini 1978c: 46)

Questi interrogativi tremendi non possono essere risolti in un mondo in cui ciascuno vive solitario il proprio dramma e allora più angosciosa si fa la tragedia degli "offesi" che nel romanzo sono rappresentati dagli ostaggi imprigionati e massacrati dai tedeschi, e dai morti fucilati in Largo Augusto: 
Chi aveva colpito non poteva colpire più nel segno. In una bambina, in un vecchio, in due ragazzi di quindici anni, in una donna, in un'altra donna. Questo era il modo migliore di colpire l'uomo. Colpirlo dove l'uomo era più debole, dove aveva l'infanzia, dove aveva la vecchiaia: dove era più uomo...

(Vittorini 1978c: 93)

E in questa dimensione di umanità indifesa è l'ignaro Giulaj sbranato dai cani tedeschi, ignaro fino all'ultimo, per una strana incoscienza, della cattiveria degli altri, dei non uomini che torturano e ammazzano.

Anche in questo romanzo così vivo di tragedia vi è l'intreccio della vicenda politica con quella sentimentale espressa in alcune pagine con una crudezza deprimente.

Se, come sottofondo, c'è sempre una problematica umana, che crea nell'opera un clima di pensosità e supera i fatti stessi, questi interrogativi rimangono tragicamente sospesi nel vuoto, non risolti perché l'autore nella sua ricerca non va oltre, o non vuole superare la constatazione di fatto.

Vittorini avvisa in una nota del suo libro: Il Sempione strizza l'occhio al Frejus del 1947 che avrebbe potuto intitolare il volume anche: "Discorso sulla morte" o "Importanza di vivere". Infatti più che ai lunghi discorsi che si svolgono tra i personaggi il lettore è teso a cogliere il senso vero di quelle considerazioni che affiorano con la pacata tristezza di chi accetta una fatalità a cui non è lecito sottrarsi.

L'ambiente in cui si svolge il romanzo è quello di una casa dove la miseria scava pareti e volti: la disoccupazione costringe sei persone a vivere col guadagno di uno solo. Il motivo dominante tra tanto squallore è quello del senso della vita e dell' attesa della morte.

Attraverso un paragone assai forzato, ma ugualmente significativo, Vittorini vuol rassomigliare l'uomo all'elefante, simbolo di una saggezza da imitare. L'autore esprime il suo pensiero sulla sorte dell'uomo che dalla vita non può attendersi molto. La felicità, chiusa nel limite del senso e della miseria, si esaurisce con una rapidità sconcertante.

Quando abbiamo trovato quel tanto che potevamo trovare, allora è finito, non c'è più nulla che ci dica qualcosa (...) non mandiamo avanti più nulla e qualunque sia la nostra età, possiamo pensare che siamo già morti. (Vittorini 1978 b: 191)

E il simbolo familiare dell'elefante si amplifica e assume significati nuovi attraverso il colloquio-soliloquio dell'operaio consumato dalla tisi che viene a passare accanto al nonno la sua ultima giornata di vita in un'estrema nostalgia di rapporto umano. Il vecchio però non sa indicargli altro che quella realtà che attende anche lui e ritorna al motivo dell'elefante:

Appena si accorgono che non mandano avanti più nulla e che sono di peso, tac, subito tagliano: si considerano morti e muoiono (...) Hanno cimiteri segreti, ignoti a loro stessi finchè sono vivi e là si recano $i$ vecchi elefanti che pensano di dover morire. Capite questo? (Vittorini 1978b:124-5) 
E questa sarà la fine che sceglierà il nonno stesso, il vecchio lavoratore del Sempione e del Frejus, in un'atmosfera di freddo stoicismo da parte sua e di indifferenza amara da parte dei suoi familiari che il problema economico ha talmente assorbito da far sentire la presenza del vecchio unicamente un peso sul bilancio familiare.

Un mattino il nonno sceglierà di concludere epicamente la sua vita e si avvierà, come aveva imparato dalla vita degli elefanti, verso il luogo segreto della sua morte.

È vestito anche con il cappotto, calzato, il cappello in testa e in mano il bastone su cui si appoggia. Come se andasse a prendere posto sulla sua sedia. (...) Il nonno si volta dalla soglia raggiunta, tra gli alberi solleva il suo bastone; con quello agitandolo, saluta.

-Siamo anche noi elefanti! Ci dice mia madre. (Vittorini 1978 b:152-3)

Lo stoicismo dei personaggi, la loro pacata rassegnazione che tiene gli occhi fissi ostinatamente a terra, richiama straordinariamente l'atmosfera del cielo dei Vinti del Verga, lo stesso fatalismo, la stessa chiusa sofferenza incide sui personaggi.

Con Le donne di Messina Vittorini tenta infine il romanzo corale e di ampio respiro, l'affresco della rinascita dell'Italia del dopoguerra.

Tutto va rifatto è necessaria ora una morale essenzialmente comunitaria che si sviluppa via via dalle forme essenziali imposte dalla convivenza più primitiva. Da qui Vittorini fa sorgere una specie di piccola comunità socialista sottomessa all'imperativo economico e a quello del clan.

In Le donne di Messina infatti Vittorini narra le vicende di un gruppo di sinistrati, di disoccupati, uomini e donne, che vanno alla ricerca di un posto al sole per lavorare e costruirsi una casa.

Il gruppo si ferma in un villaggio distrutto sulla linea gotica ed incominciano insieme il lavoro di ricostruzione.

Il superamento del proprio io e l'adeguarsi alle esigenze della vita comunitaria è la meta suprema della nuova etica. L'atmosfera tipica di questo romanzo è quindi eminentemente corale: c'è la nuova società in contrasto con la vecchia conservatrice della legalità, del conformismo e della burocrazia.

L'amore che lega i due protagonisti: Ventura, exfascista presente al villaggio in incognita e Siracusa, la ragazza venuta al Nord con le truppe dei vincitori, è il filone centrale del romanzo ed ha un epilogo tragico. Ventura, prima di essere fucilato, perché scoperto fascista, esorta gli abitanti del nuovo ipotetico villaggio a non abbandonare quell'oasi di libertà assoluta, non condizionata da alcun obbligo verso la società alla quale egli attribuisce tuttte le colpe e le responsabilità dell'annullamento della personalità umana.

Egli stesso però, che in un accesso di furore, ha ucciso Siracusa, incarna nella nuova società il trionfo del male e l'offesa dell'uomo, i vecchi tarli dell'infelicità dell'uomo.

Il romanzo, che avrebbe voluto essere polemico nei confronti della società, presenta le pecche ormai consuete nella produzione vittoriana, soprattutto risente ancora una volta che le esigenze di riscatto che Vittorini proclama nei confronti dell'uomo e della società si isteriliscono in forme velleitarie senza una vera forza di redenzione.

Per concludere certamente Vittorini ha avuto influenza sul suo tempo perché interpretava un senso di disagio e di sofferenza comune a tutti. 
Ogni sua opera più significativa, infatti, è segnata dai problemi sociali e politici del momento: l'avvento al potere del fascismo in Il garofano rosso, la guerra di Spagna in Conversazione in Sicilia, la resistenza in Uomini e no, il disagio del dopoguerra in Il Sempione strizza l'occhio al Frejus e in Le donne di Messina.

Egli stesso sottolinea questa partecipazione ai problemi del suo tempo:

Io invidio gli scrittori che hanno capacità di restare interessati al proprio lavoro mentre infuriano pestilenze e guerre: Joyce che continuò a scrivere l'Ulisses durante la guerra del '14 '18 (...) Un grosso evento pubblico può distrarmi, purtroppo, e provocare un mutamento di interessi nel mio lavoro come né più né meno una mia sventura (o ventura) personale. (Pautasso 1967: 85)

Questa partecipazione sofferta ha dato a Vittorini l'alone di guida morale delle giovani generazioni.

Egli aveva scritto nella prefazione a Il garofano rosso:

Io scrivo perché ho una verità da dire e se torno a scrivere non è perché mi accorgo di altre verità che si possono aggiungere, ma perché qualcosa che continua a mutare nella verità mi sembra esigere che non si smetta mai di dirla. (Vittorini 1981: 197)

Ma qual è questa verità che egli sostiene? È la verità del "mondo offeso."

I suoi romanzi, a volte violentemente realisti, rimangono sempre in un clima di sospensione e di amarezza.

Il suo itinerario senza speranza il colloquio che Vittorini insistentemente vorrebbe instaurare con l'umanità sofferente rimane monco, anzi si esaurisce in una smarrita enunciazione di dubbi e di interrogativi.

La considerazione verso l'umanità più diseredata e sofferente, la capacità di considerarla, con occhio di predilezione e di rispetto, la recriminazione contro il sistema di una società che deve essere rifatta vivono, in prima persona, in Vittorini scrittore e uomo.

\section{Bibliografía}

Pautasso, Sergio. 1967. Elio Vittorini. Torino: Borla.

Pazzaglia, Mario. 1972. Gli Autori della letteratura italiana. Bologna: Zanichelli.

Ricciardi, Mario. 1989. La Letteratura in Italia. Milano: Bompiani.

Scaramucci, Italo. 1956. Romanzi del nostro tempo. Brescia: ed. La Scuola.

Vittorini, Elio. 1945. "Politecnico”. I.

Vittorini, Elio. 1957. Diario in pubblico. Milano: Bompiani. 
Vittorini, Elio. 1964. Le donne di Messina. Milano: Bompiani.

Vittorini, Elio. 1978a. Conversazione in Sicilia. Torino: Einaudi.

Vittorini, Elio. 1978b. Il Sempione strizza l'occhio al Frejus. Milano: Bompiani.

Vittorini, Elio. 1978c. Uomini e no. Milano: Mondadori.

Vittorini, Elio. 1981. Il garofano rosso. Milano: Mondadori.

Vittorini, Elio. 1986. Picciola borghesia. Milano: Mondadori. 